Article

\title{
Optical Properties of Electrically Active Gold Nanoisland Films Enabled with Interfaced Liquid Crystals
}

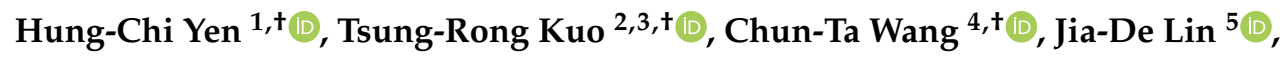 \\ Chia-Chun Chen ${ }^{1}$ and Yu-Cheng Hsiao ${ }^{3,6, *}$ \\ 1 Department of Chemistry, National Taiwan Normal University, 88 Ting-Chow Rd., Sec. 4, Taipei 11677, \\ Taiwan; hungchiyen@gmail.com (H.-C.Y.); cjchen@ntnu.edu.tw (C.-C.C.) \\ 2 Graduate Institute of Nanomedicine and Medical Engineering, Taipei Medical University, 250 Wuxing St., \\ Taipei 11031, Taiwan; trkuo@tmu.edu.tw \\ 3 International PhD Program for Biomedical Engineering, Taipei Medical University, 250 Wuxing St., \\ Taipei 11031, Taiwan \\ 4 Department of Photonics, National Sun Yat-Sen University, 70 Lienhai Rd., Kaohsiung 80424, Taiwan; \\ wangchunta4@gmail.com \\ 5 Department of Engineering Science, University of Oxford, Parks Road, Oxford OX1 3PJ, UK; \\ geman1218@yahoo.com.tw \\ 6 Graduate Institute of Biomedical Optomechatronics, Taipei Medical University, 250 Wuxing St., Taipei 11031, \\ Taiwan \\ * Correspondence: ychsiao@tmu.edu.tw \\ + These authors contributed equally to this work.
}

Received: 25 November 2019; Accepted: 4 February 2020; Published: 9 February 2020

check for updates

\begin{abstract}
A system comprising a gold nanoisland film (Au NIF) covered with a liquid crystal (LC) material is introduced. By applying a voltage across the LC bulk, we demonstrate that changes in the refractive-index and orientation significantly modified the hybrid plasmonic-photonic resonances of the Au NIF. The hybrid structure enabled active control of the spectrum of the resonance wavelength of the metallic nanoisland by means of an externally applied electric field. Our modeling supports the observed results in LC/Au NIF. In a combination of the nanostructured surface with birefringent LCs, nonpolarized wavelength tunability of $\sim 15 \mathrm{~nm}$ and absorbance tunability of $\sim 0.024$ were achieved in the visible wavelength, opening the door to optical devices and nanoscale sensors.
\end{abstract}

Keywords: liquid crystal; optical device; gold nanoisland film

\section{Introduction}

Gold nanoisland films (Au NIFs) with nanosized gaps have been fabricated for applications in imaging and sensing due to their unique surface plasmon resonances [1-3]. Au NIFs with surface plasmon resonances in the near-infrared (NIR) region have been utilized to enhance the fluorescence intensity of various NIR fluorophores for imaging and sensing applications. For example, biochips of Au NIFs captured immunoglobulin G (IgG) and IgA antibodies against Zika virus or dengue virus antigens in human serum and then were conjugated with fluorophores of anti-human IgG-IRDye680 and anti-human IgA-IRDye800 [4]. An Au NIF platform showed the ability to amplify the NIR fluorescence of fluorophores of anti-human IgG-IRDye680 and anti-human IgA-IRDye800 by about 100-fold, allowing for highly sensitive detection of Zika virus infection and dengue virus infection. Although $\mathrm{Au}$ NIFs have been extensively applied for imaging and sensing applications, the development of Au NIFs of a suitable wavelength and intensity of surface plasmon resonance is still being processed to 
optimize detection conditions. It is practically significant to enable active control of these plasmon resonance properties by external tuning methods. Such tunability can be achieved by applying voltage, heat, or an illumination profile after incorporating other materials [5-7]. Recently, the electrical tuning of nano-antenna resonances in the visible regime was demonstrated [8].

Liquid crystals (LCs) are interesting materials because their electrically induced reorientation of molecules can modify the resonance conditions of optical resonators. Indeed, LCs can be employed to control resonances of metallic nanostructures, including localized surface plasmon resonance (LSPR)-based nano-antennas and surface plasmon polaritons in metallic films [9-12]. In addition, many plasmonic response combined with LC devices have been proposed in the past [13-15]. To the best of our knowledge, a hybrid LC/nanoisland structure has not been reported until now. The tuning ranges of LC-based metallic nanostructures are quite limited in polarized light. However, polarization-independent LC-based plasmonic surfaces have been invented with a large range of the colour spectrum [16]. In addition, the large tuning range of hybrid LC/metallic nanostructures are also proposed in the literature [17].

In this study, we first show the active tuning of Au NIF resonances in a metallic nanostructure embedded in an LC matrix. Tuning was achieved by applying an electric field to the LC/Au NIF device confined by an Au nanostructured substrate and a conducting glass substrate coated with polyimide (PI) for planar alignment of the LC molecules. As evidenced by the voltage-dependent nonpolarized transmission spectrum, a shift in the optical resonance of $15 \mathrm{~nm}$ was achieved.

\section{Materials and Methods}

All chemicals were obtained from suppliers and used without further purification. Ammonium hydroxide $\left(\mathrm{NH}_{4} \mathrm{OH}, 30 \%\right)$, hydroxylamine hydrochloride $\left(\mathrm{NH}_{2} \mathrm{OH} \cdot \mathrm{HCl}, 98 \%\right)$, polyacrylic acid (PAA, Mw: 100k, 35 wt. \% in $\left.\mathrm{H}_{2} \mathrm{O}\right)$, and sodium borohydride $\left(\mathrm{NaBH}_{4}, 98 \%\right)$ were purchased from Sigma-Aldrich (St. Louis, MO, USA). Dimethylformamide (DMF, 99.5\%) and hydrogen tetrachloroaurate(III) trihydrate $\left(\mathrm{HAuCl}_{4} \cdot 3 \mathrm{H}_{2} \mathrm{O}\right)$ were purchased from Alfa Aesar (Ward Hill, MA, USA). Fluorine doped tin oxide (FTO) substrates were purchased from STAREK Scientific Co., Ltd. (Taipei, Taiwan (R.O.C.)). To fabricate Au NIFs, the FTO substrates with a size of $3 \times 3 \mathrm{~cm}$ were first rinsed with pure water, and then ultrasonically cleaned in acetone, methanol, and pure water for $10 \mathrm{~min}$ in each solvent. Then, a carboxylic acid-rich polymer of PAA was pre-coated onto the substrates to improve the adhesion of Au seeds to the FTO substrates. Typically, $200 \mu \mathrm{L}$ of DMF solution containing PAA $(0.7 \% w / w)$ was dropped onto a FTO substrate, followed by spin coating at $1500 \mathrm{rpm}$ for $20 \mathrm{~s}$. The speed was subsequently increased to $2500 \mathrm{rpm}$ for $180 \mathrm{~s}$. Afterwards, the FTO substrate with the PAA coating was annealed in an air atmosphere at $180^{\circ} \mathrm{C}$ for $1 \mathrm{~h}$. To generate the $\mathrm{Au}$ seed layer, the FTO substrate with the PAA coating was immersed in a $45 \mathrm{~mL}$ mixture containing $\mathrm{HAuCl}_{4}(3 \mathrm{mM})$ and $\mathrm{NH}_{4} \mathrm{OH}(0.3 \mathrm{M})$ at $10{ }^{\circ} \mathrm{C}$ for $20 \mathrm{~min}$ to deposit $\mathrm{Au}^{3+}$ ions. After rinsing with pure water, the FTO substrate was immersed in a $\mathrm{NaBH}_{4}(1 \mathrm{mM})$ solution at $10{ }^{\circ} \mathrm{C}$ for 2 min to reduce the adsorbed $\mathrm{Au}^{3+}$ ions to $\mathrm{Au}$ seeds. To promote the growth of the Au NIF, the FTO substrate with the Au seed layer was immersed in $50 \mathrm{~mL}$ of a growth solution containing $\mathrm{HAuCl}_{4}(0.5 \mathrm{mM})$ and $\mathrm{NH}_{2} \mathrm{OH}(0.5 \mathrm{mM})$ at $30^{\circ} \mathrm{C}$ for $5 \mathrm{~min}$. After washing with pure water, the FTO substrate with the Au NIF was subsequently blow-dried with nitrogen and stored at room temperature in a vacuum and dark environment for further measurements. SEM images of the Au NIF were acquired on a field emission SEM (S-4800, Hitachi Ltd., Tokyo, Japan) with an acceleration voltage of $10 \mathrm{kV}$. An arbitrary function generator (Tektronix AFG-3022B, Beaverton, OR, USA) was also employed to apply various electrical frequency-modulated voltages. In addition, transmission spectra of the LC/Au NIFs were obtained with a fiberoptic spectrometer (Ocean Optics HR $2000^{+}$, Ocean Optics, Largo, FL, USA). All experimental data were acquired at $26 \pm 1{ }^{\circ} \mathrm{C}$. 


\section{Results and Discussion}

Figure $1 \mathrm{a}, \mathrm{b}$ shows scanning electron microscopic (SEM) images and the corresponding magnified region of $\mathrm{Au}$ NIFs fabricated on a FTO coated glass substrate. The Au NIF was fabricated with a facile seed-mediated growth approach in the solution phase. Au NIFs with elongated nanostructures and winding inter-island gaps were characterized by SEM, and the average nanoisland size and gap distance were calculated from SEM images using ImageJ software. Built-in functions of particle analysis and watershed segmentation were used to determine the average island size and gap distance (Figure 1c,d). The Au NIF revealed an average nanoisland size of $6.6 \times 10^{4} \pm 1.8 \times 10^{4} \mathrm{~nm}^{2}$ and an average inter-island gap distance of $69.3 \pm 32.1 \mathrm{~nm}$.

(a)

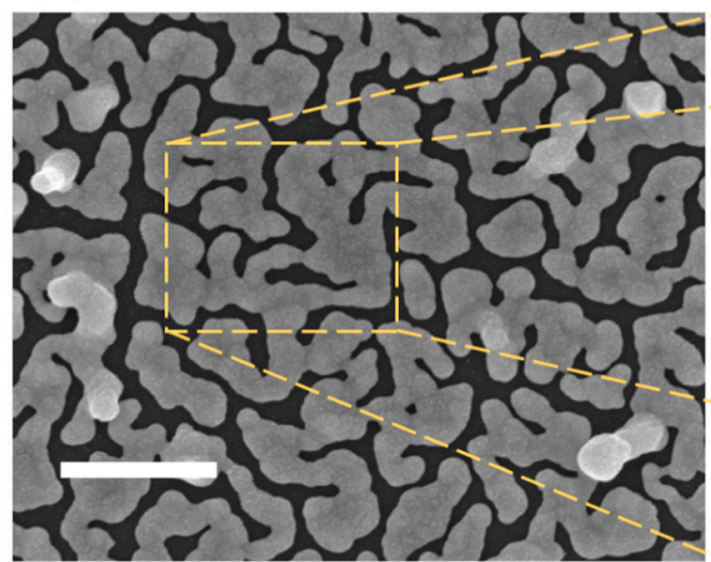

(c)

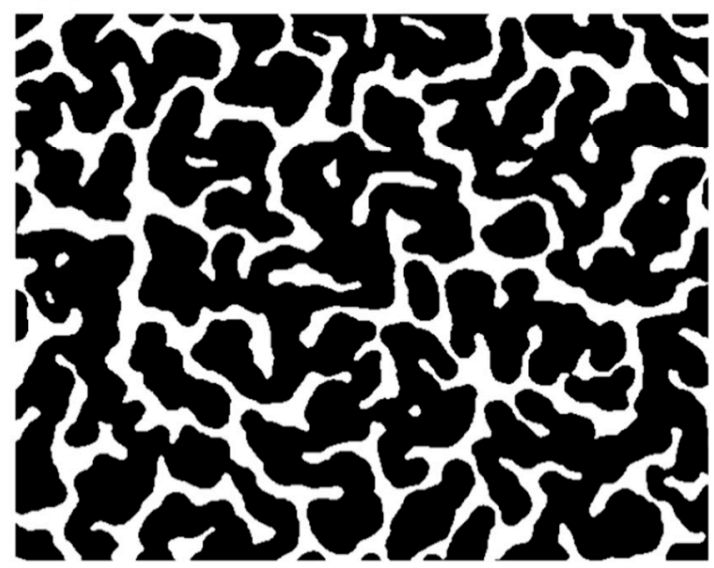

(b)

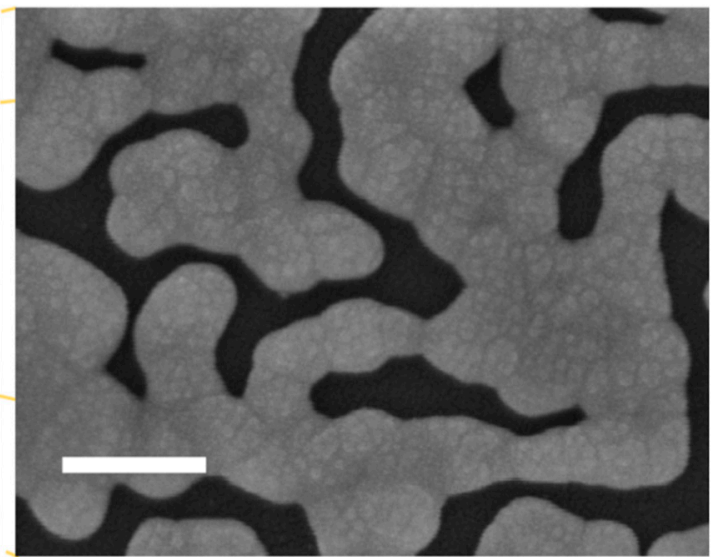

(d)

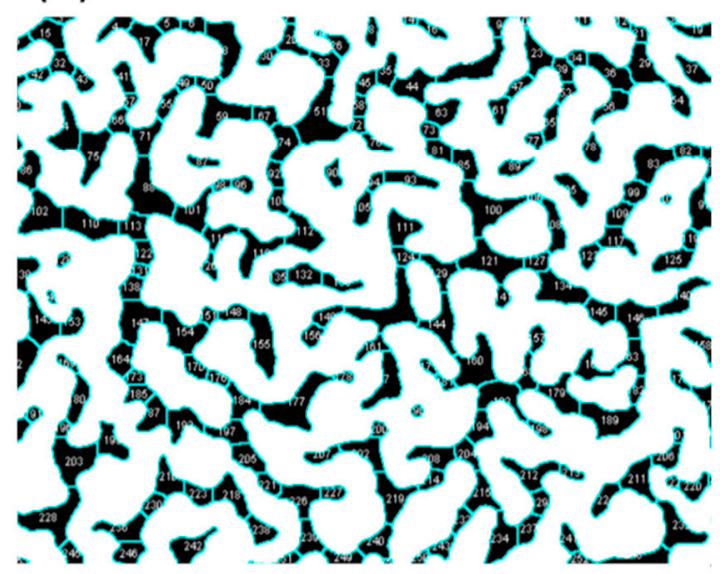

Figure 1. (a) SEM image of the gold nanoisland film (Au NIF) (scale bar = $500 \mathrm{~nm}$ ) and (b) the selected magnified area (scale bar $=150 \mathrm{~nm}$ ). (c) The converted binary image of the Au NIF used for the calculation of average nanoisland size from (a). (d) An inverted image of (c) was applied to determine the average inter-island gap distance, where the blue line shows gaps after the watershed segmentation process.

The substrate with the nanostructured surface served as the bottom substrate for the LC cell as schematically shown in Figure 2. The other substrate was a typical FTO-coated glass slide spin-coated with a PI alignment layer and treated with mechanical buffing. The assembled cell had a thickness of $\sim 10 \mu \mathrm{m}$, as determined by silica spacers. E7 LC material was introduced into the empty cell. The top and bottom electrodes permit AC voltage to be applied across the cell thickness. Nonpolarized white light was successively transmitted through the top glass substrate and LC layer, and then was coupled to the Au NIF with plasmonic resonance. The LSPR can be adjusted by the dielectric constant of the 
surrounding LC material which is determined by the reorientation of the LC director by the externally applied electric field. Figure 3 shows optical textures of the tunable LC/Au NIF device with various applied voltages under crossed polarizers. We observed that the optical textures of the LC/Au NIFs were in a planar arrangement at $0 \mathrm{~V}$. The LC/Au NIF textures were dark and bright when the angle between the rubbing direction and the polarizers was 0 and 45 , respectively. When we increased the applied voltage, the direction of the LC molecules tended to reorient toward the direction of the electric field, and the colors of the optical textures gradually changed. The brightness slowly dimmed because of the decreasing birefringence.

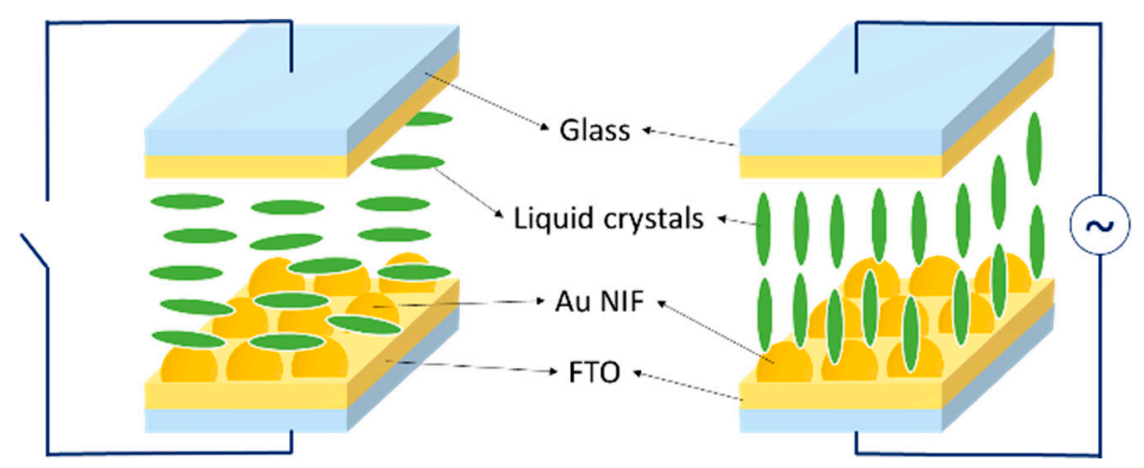

Figure 2. Schematic of the liquid crystal (LC)/gold nanoisland film (Au NIF) devices. An applied electric field across the cell causes the LC to reorient and changes the wavelengths of the resonant light.

OV

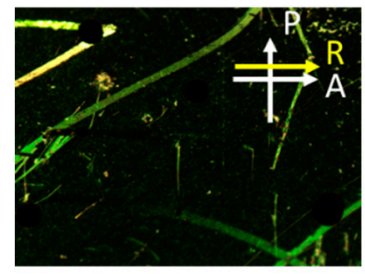

$3 \mathrm{~V}$

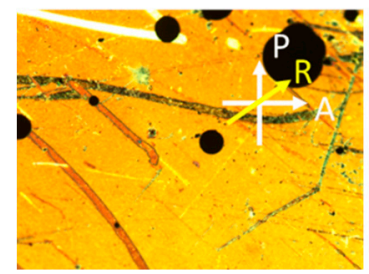

OV

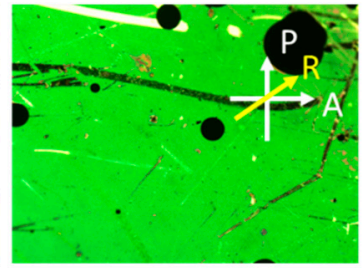

7V

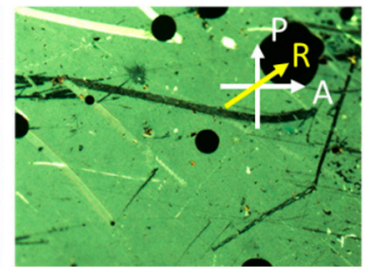

2V

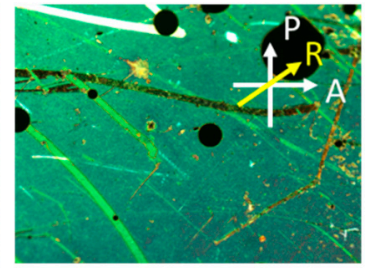

$8 V$

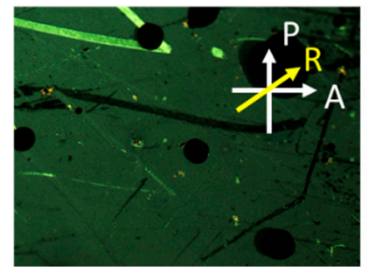

Figure 3. Optical textures of tunable liquid crystal (LC)/gold nanoisland film (Au NIF) with various applied voltages under crossed polarizers. White arrows indicate the transmission axes of the polarizer and analyzer, while the yellow arrow indicates the rubbing direction.

In addition, the experimentally measured nonpolarized absorption spectrum of the LC/Au NIF as a function of applied voltages is shown in Figure 4. In Figure 4a, one can observe a relationship between the applied voltage and resonant wavelength in an AC field. A greater voltage was required to reorient the LC molecules near the nanostructure due to the strong anchoring forces of the nanostructure. That is why the tuning range of the active plasmonic device was limited in the past. However, we used the Au NIF which has a spherical bulge. With the random spherical shape of the Au NIF, the LC molecules reduced the arrangement of the groove effect. Consequently, the direction of the LC molecules could more easily be tilted up by applying an electrical field. The interface effect between gold and LC molecules is studied in the literature [17]. In Figure 4a, when the incident light went through the planar LC layer at $0 \mathrm{~V}$, the effective refractive index near the Au NIF experienced by the nonpolarized incident 
light was around $\left(n_{e}+n_{0}\right) / 2$, and the resonant wavelength was $477 \mathrm{~nm}$. The LC molecule tilted under applied voltages of $2,4,6$, and $8 \mathrm{~V}$, and the resonant wavelength was red shifted. When the applied voltage was $8 \mathrm{~V}$, the LC molecules almost completely reoriented themselves in a direction normal to the substrate surface, and the effective refractive index near the Au NIF was close to the ordinary refractive index, $\mathrm{n}_{\mathrm{o}}$. Simulation results in previous research also showed that the resonant wavelength increased with a decreasing reflective index [18]. The curvature of LSPR to refractive index changes by using Mie's theory, as well as the analytical expression of the LSPR curvature to the refractive index is proposed theoretically in the literature [19]. However, the resonant wavelength did not change when the applied voltage exceeded $8 \mathrm{~V}$, because a quasi-homotropic $\mathrm{LC}$ was exhibited when the voltage exceeded 8 V. Moreover, the absorbance of the Au NIF decreased with increasing electrical voltage, because of a reduction in the refractive index. Spectra of the LC/Au NIFs with applied DC voltages of amplitudes $0 \sim 8 \mathrm{~V}$ are shown in Figure $4 \mathrm{~b}$. Both the wavelength and absorbance tunability of the $\mathrm{LC} / \mathrm{Au}$ NIFs were not obvious under DC operation, because DC voltage possesses an ionic effect, which makes LC molecules insensitive to the applied voltage [20]. In the past, the birefringence change of LC induced resonance shifting is not significant. However, the spherical Au structure makes LCs easier to change arrangement nearing the interface and to shift the resonance.
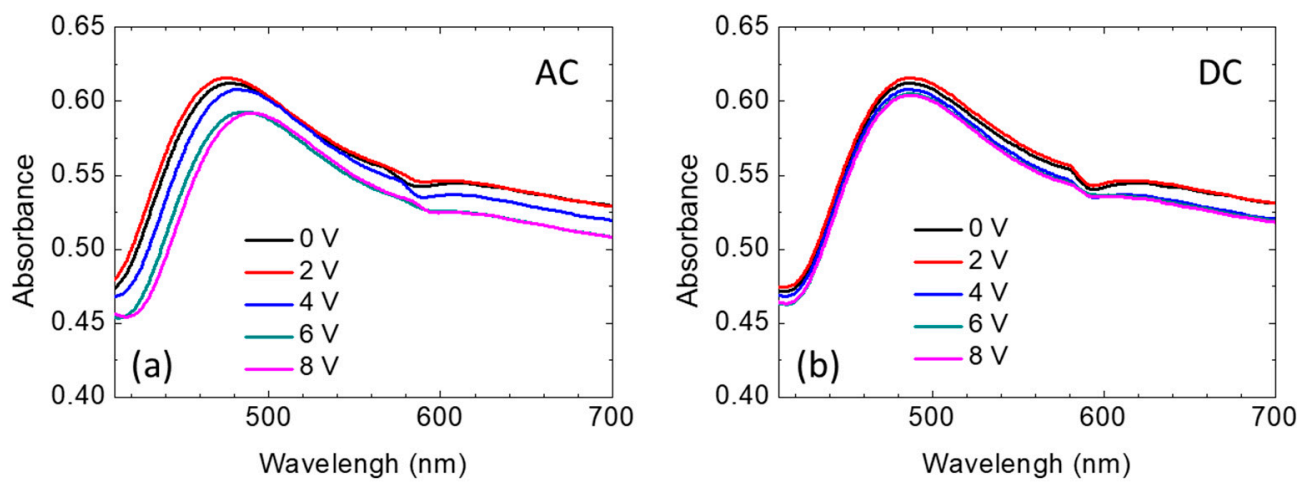

Figure 4. Nonpolarized spectra of the liquid crystal (LC)/gold nanoisland film (Au NIF) with applied voltages of amplitudes $0 \sim 8 \mathrm{~V}$ under (a) Alternating current (AC) and (b) Direct current (DC) voltage.

Maxwell's equation is solvable for spherical particles, as first proposed by Gustav Mie in 1908, and further developed by Bohren and Huffman. Thus, the absorption and scattering of spherical particles can be calculated from Mie's theory [21]. The wavelength- and refractive index-dependent absorption A $(\lambda, n)$ of the LC/Au NIF can be derived from Mie's theory:

$$
A(\lambda, n)=\frac{18 \pi V n_{e f f}^{2} N l}{\lambda} \frac{\left(a_{2}+b_{2} \lambda\right)}{\left(a_{2}+b_{2} \lambda\right)^{2}\left(b_{1}+a_{1}+2 n_{e f f}{ }^{2}\right)+1}
$$

where $a_{1}, a_{2}, b_{1}, b_{2}$, and $\pi$ are constants, $V$ is the volume of the particle, $N$ is the density of electrons, $\lambda$ is the wavelength of light, $l$ is the length of the optical path, and $n_{\text {eff }}$ is the effective refractive index of the surrounding medium. In addition, the effective refractive index $\left(n_{\text {eff }}\right)$ of the surrounding medium, which consisted of LC molecules in this case, can be described by:

$$
n_{e f f}(\theta)=n_{e} \sin ^{2} \theta+n_{o} \cos ^{2} \theta
$$

where $\theta$ is the angle between the direction of the LCs and the direction of the incident light. Based on the equation, the effective refractive index is sensitive to the tilting angle of the LCs. According to these equations, the absorption of the LC/Au NIF decreases with a reduction in the effective refractive index (or increasing applied voltage), which is the same behavior as in Figure $4 \mathrm{a}, \mathrm{b}$. Figure $5 \mathrm{a}, \mathrm{b}$ shows the absorbance and wavelength tuning of the LC/Au NIFs with various applied voltages of amplitudes $0 \sim 8 \mathrm{~V}_{\text {rms }}$. From Figure $5 \mathrm{a}$, one can see that the absorbance was highest at $2 \mathrm{~V}_{\mathrm{rms}}$, because the LC 
molecules are better aligned under a small applied voltage. The greatest portion of $\left(n_{e}+n_{o}\right) / 2$ of the LCs surrounding the $\mathrm{Au}$ NIF is demonstrated. The value of $\left(n_{e}+n_{0}\right) / 2$ gradually changed to $\mathrm{n}_{\mathrm{o}}$, resulting in a decrease in the absorbance and a red shift of the LSPR wavelengths. Based on the equations, the tilting angles of the LCs (complementary to angle $\theta$ ) had a great influence on the absorbance and wavelength of LC/Au NIFs. Dynamic measurements of the tilting angle, namely, the polar angle measured from the substrate plane, were carried out using a typical crystal-rotation method [22]. Figure 6 displays variations in the tilting angle of the LC/Au NIFs with applied voltages measured by the typical crystal-rotation method. The angle dramatically increased from $\sim 15$ to $\sim 80^{\circ}$ and was finally saturated at $\sim 80^{\circ}$. Compared to the well-known LC plasmon resonance device, the most advantageous feature of our LC/Au NIF is the nonpolarized, tunable LSPR. We found that the wavelength tunability of $\sim 15 \mathrm{~nm}$ and absorbance tunability of $\sim 0.024$ were shown based on the LC/Au NIFs.
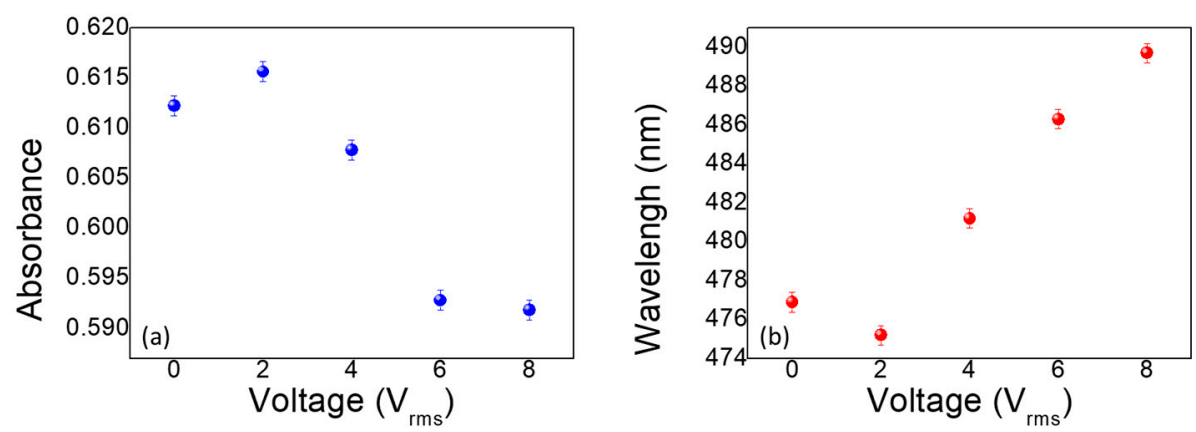

Figure 5. Absorbance and peak wavelength of the liquid crystal (LC)/gold nanoisland film (Au NIF) with various applied voltages of amplitudes $0 \sim 8 \mathrm{~V}_{\text {rms. }}$.

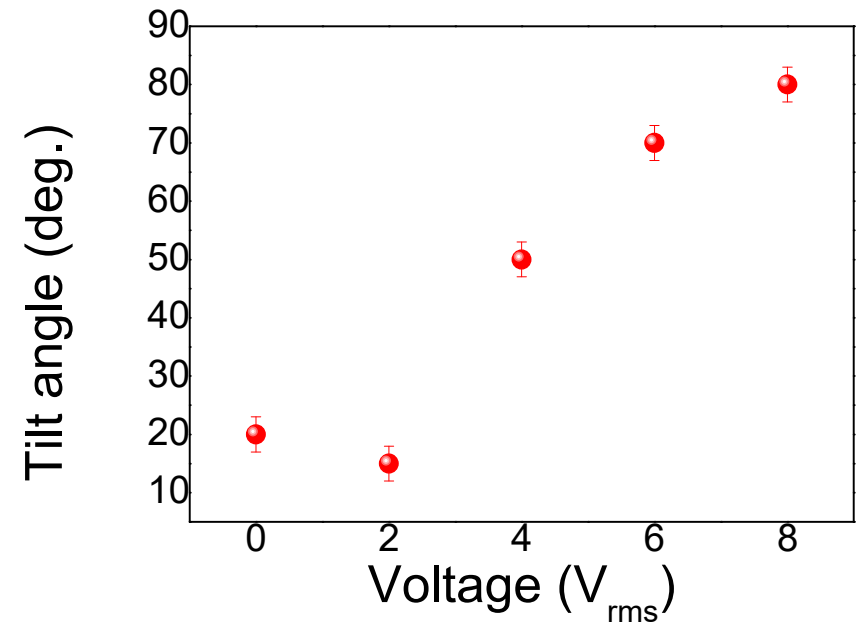

Figure 6. Variation of the tilting angle of the liquid crystal (LC)/gold nanoisland film (Au NIF) with various applied voltages.

\section{Conclusions}

In summary, optical properties of the Au NIFs covered with LC materials are introduced. We demonstrated that the resonance wavelength-dependent refractive index and orientation of the LCs in the hybrid LC/Au NIFs significantly changed when various voltages were applied. The hybrid $\mathrm{LC} / \mathrm{Au}$ NIF structure enabled the active control of the resonance wavelength of the spectrum in the metallic nanoisland. A combination of the nanostructured surface and birefringent LC opens the door for new electro-optical devices, tunable devices, nanoscale sensors, and additional applications.

Author Contributions: Data curation, H.-C.Y., C.-C.C., C.-T.W., and T.-R.K.; funding acquisition, H.-C.Y. and Y.-C.H.; investigation, H.-C.Y. and Y.-C.H.; project administration, Y.-C.H.; writing, Y.-C.H. and J.-D.L. All authors have read and agreed to the published version of the manuscript. 
Funding: This work was financially supported by the Ministry of Science and Technology, Taiwan, under grant numbers MOST 108-2636-E-038-001 and MOST 108-2636-E-110-002 and by Taipei Medical University, Taiwan, under grant number TMU106-AE1-B49.

Conflicts of Interest: The authors declare no conflict of interest.

\section{References}

1. Hong, G.; Wu, J.Z.; Robinson, J.T.; Wang, H.; Zhang, B.; Dai, H. Three-dimensional imaging of single nanotube molecule endocytosis on plasmonic substrates. Nat. Commun. 2012, 3, 700. [CrossRef] [PubMed]

2. Zhang, B.; Price, J.; Hong, G.; Tabakman, S.M.; Wang, H.; Jarrell, J.A.; Feng, J.; Utz, P.J.; Dai, H. Multiplexed cytokine detection on plasmonic gold substrates with enhanced near-infrared fluorescence. Nano Res. 2013, 6, 113-120. [CrossRef]

3. Zhang, B.; Kumar, R.B.; Dai, H.; Feldman, B.J. A plasmonic chip for biomarker discovery and diagnosis of type 1 diabetes. Nat. Med. 2014, 20, 948-953. [CrossRef] [PubMed]

4. Zhang, B.; Pinsky, B.A.; Ananta, J.S.; Zhao, S.; Arulkumar, S.; Wan, H.; Sahoo, M.K.; Abeynayake, J.; Waggoner, J.J.; Hopes, C. Diagnosis of Zika virus infection on a nanotechnology platform. Nat. Med. 2017, 23, 548. [CrossRef]

5. Jun, Y.C.; Huang, K.C.Y.; Brongersma, M.L. Plasmonic beaming and active control over fluorescent emission. Nat. Commun. 2011, 2, 283. [CrossRef]

6. Shadrivov, I.V.; Kapitanova, P.V.; Maslovski, S.I.; Kivshar, Y.S. Metamaterials controlled with light. Phys. Rev. Lett. 2012, 109, 083902. [CrossRef]

7. Lumdee, C.; Toroghi, S.; Kik, P.G. Post-fabrication voltage controlled resonance tuning of nanoscale plasmonic antennas. ACS Nano 2012, 6, 6301-6307. [CrossRef]

8. Abass, A.; Rodriguez, S.R.K.; Ako, T.; Aubert, T.; Verschuuren, M.; van Thourhout, D.; Beeckman, J.; Hens, Z.; Rivas, J.G.; Maes, B. Active liquid crystal tuning of metallic nanoantenna enhanced light emission from colloidal quantum dots. Nano Lett. 2014, 14, 5555-5560. [CrossRef]

9. Kossyrev, P.A.; Yin, A.; Cloutier, S.G.; Cardimona, D.A.; Huang, D.; Alsing, P.M.; Xu, J.M. Electric field tuning of plasmonic response of nanodot array in liquid crystal matrix. Nano Lett. 2005, 5, 1978-1981. [CrossRef]

10. Dickson, W.; Wurtz, G.A.; Evans, P.R.; Pollard, R.J.; Zayats, A.V. Electronically controlled surface plasmon dispersion and optical transmission through metallic hole arrays using liquid crystal. Nano Lett. 2008, 8, 281-286. [CrossRef]

11. Khatua, S.; Chang, W.-S.; Swanglap, P.; Olson, J.; Link, S. Active modulation of nanorod plasmons. Nano Lett. 2011, 11, 3797-3802. [CrossRef] [PubMed]

12. Dridi, M.; Vial, A.J. FDTD modeling of gold nanoparticles in a nematic liquid crystal: Quantitative and qualitative analysis of the spectral tunability. Phys. Chem. C 2010, 114, 9541-9545. [CrossRef]

13. Hsiao, V.K.; Zheng, Y.B.; Juluri, B.K.; Huang, T.J. Light-driven plasmonic switches based on au nanodisk arrays and photoresponsive liquid crystals. Adv. Mater. 2008, 20, 3528-3532. [CrossRef]

14. Lu, M.; Zhang, X.; Liang, Y.; Li, L.; Masson, J.F.; Peng, W. Liquid crystal filled surface plasmon resonance thermometer. Opt. Express 2016, 24, 10904-10911. [CrossRef] [PubMed]

15. Cetin, A.E.; Mertiri, A.; Huang, M.; Erramilli, S.; Altug, H. Thermal tuning of surface plasmon polaritons using liquid crystals. Adv. Opt. Mater. 2013, 1, 915-920. [CrossRef]

16. Franklin, D.; Chen, Y.; Vazquez-Guardado, A.; Modak, S.; Boroumand, J.; Xu, D.; Wu, S.-T.; Chanda, D. Polarization-independent actively tunable colour generation on imprinted plasmonic surfaces. Nat. Commun. 2015, 6, 7337. [CrossRef]

17. Hsiao, Y.-C.; Su, C.-W.; Yang, Z.-H.; Cheypesh, Y.I.; Yang, J.-H.; Reshetnyak, V.Y.; Chen, K.-P.; Lee, W. Electrically active nanoantenna array enabled by varying the molecular orientation of an interfaced liquid crystal. RSC Adv. 2016, 6, 84500-84504. [CrossRef]

18. Schindelin, J.; Arganda-Carreras, I.; Frise, E.; Kaynig, V.; Longair, M.; Pietzsch, T.; Preibisch, S.; Rueden, C.; Saalfeld, S.; Schmid, B. Fiji: An open-source platform for biological-image analysis. Nat. Methods 2012, 9 , 676-682. [CrossRef]

19. Chen, P.; Liedberg, B. Curvature of the localized surface plasmon resonance peak. Anal. Chem. 2014, 86, 7399-7405. [CrossRef] 
20. Hsiao, Y.-C.; Huang, S.-M.; Yeh, E.-R.; Lee, W. Temperature-dependent electrical and dielectric properties of nematic liquid crystals doped with ferroelectric particles. Displays 2016, 44, 61-65. [CrossRef]

21. Mie, G. Beiträge zur Optik trüber Medien, speziell kolloidaler Metallösungen. Ann. Phys. 1908, 330, $377-445$. [CrossRef]

22. Gwag, J.S.; Lee, S.H.; Park, K.-H.; Park, W.S.; Han, K.-Y.; Jhun, C.G.; Yoon, T.-H.; Kim, J.C.; Song, D.; Shin, D. Simple method for measuring the high pretilt angle of nematic liquid crystals. J. Appl. Phys. 2003, 93, 4936. [CrossRef]

(C) 2020 by the authors. Licensee MDPI, Basel, Switzerland. This article is an open access article distributed under the terms and conditions of the Creative Commons Attribution (CC BY) license (http://creativecommons.org/licenses/by/4.0/). 